\title{
Development and Design of Forensic Expert System in Bridge Engineering
}

Li Lin ${ }^{1, a}$, Dandan Xia ${ }^{2, b}$, Haitao $\mathrm{Hu}^{3, \mathrm{c}}$, Xiangmin Zhang ${ }^{4, \mathrm{~d}}$, Fuqiang $\mathrm{He}^{5, \mathrm{e}}$

${ }^{1}$ School of Civil \& Architecture Engineering, Xiamen University of Technology, Xiamen, Fujian, 361024, College of Civil Engineering, Hunan University, Changsha, Hunan, 410082, China

${ }^{2}$ School of Civil \& Architecture Engineering, Xiamen University of Technology, Xiamen, Fujian, 361024, China, Industrial Systems Engineering, University of Regina, Regina, SK, S4S OA2, Canada

${ }^{3}$ School of Civil \& Architecture Engineering, Xiamen University of Technology, Xiamen, Fujian, 361024, China

${ }^{4}$ School of Civil \& Architecture Engineering, Xiamen University of Technology, Xiamen, Fujian, 361024, China

${ }^{5}$ School of Civil \& Architecture Engineering, Xiamen University of Technology, Xiamen, Fujian, 361024, China, Scientific and Technological Innovation Platform of Fujian Province, Xiamen University, Xiamen, Fujian, 361005, China

a2011110904@xmut.edu.cn, ${ }^{\text {bxia229@uregina.ca. }{ }^{c} 2013120901 @ x m u t . e d u . c n .{ }^{d} 2012120901 @ x m ~}$ ut.edu.cn, ${ }^{\mathrm{e}} 2011110901 @ x m u t . e d u . c n$

Keywords: Bridge Engineering; Forensic Expert system, Neural Network, Engineering Forensic; System model.

Abstract. Considering the complication of impact factor in the bridge engineering identification, it is regarded as an inverse problem and the neural network method is applied to avoid the difficulty caused by the uncertainty of the system. The data model and neural network simulation model of the bridge engineering system are set with the application of VFP and MATLAB respectively. By the positive connection of the two key models and other assisted model, the collection of data, simulation of system and identification analysis are conducted to provide an open, friendly and safe platform for the completion of information and the network practice.

\section{Introduction}

The accident forensic of the bridge construction is to find the reason and responsibility of the accident through the analysis of the damage. It is to analyze the procedure and failure mechanism of the accident by the structural level to obtain the composition of the maximum risk, which can be regarded as an inverse problem. According to the general model, the accident can be concluded as "reason signals" [1] and the forensic result can be defined as "result signals". However, the reason-result model can include all procedure such as civilization, design, construction and operation, and too many participants and complicated crafts are involved [2]. The system therefore became a classical gray system. Therefore, it is necessary to solve the gray system to obtain a more accurate forensic system. With the usage of neutral network, the forensic expert system are modelled to analyze the connection of damage of the construction and responsibility risk, to ensure higher accuracy and stability of the results analyzed by the forensic expert system.

\section{Solution Comparison of Forensic Problem}

As a classical gray inverse problem, the main difficulty of solving forensic problem is applicability. It is hard to get the deterministic solution of gray system with only differential equation. The additional conditions are needed. Moreover, even though the additional conditions can determine the analytical solution, the applicability should also be solved. The general mathematic model is as follow [3, 4]: 
Assuming that the $\mathrm{F}$ and $\mathrm{U}$ are matric space (solution space and data space respectively), the operator $A$ : $F-U$, then the inverse problem can be written as sub equation

$$
\mathrm{A} z=u,(z \in F, u \in U)
$$

In the equation, $\mathrm{A}$ is the integral operator, differential operator or matrix. It is a forward problem to know $A, z$ and solve $u$. However, it is an inverse problem to know $u$ and solve $A$ or $z$. Equation (1) is the general mathematic model of inverse problem.

In the equation (1), assuming that $\rho F$ and $\rho U$ are the measurements of the space $F$ and $U$ respectively, the operator $A$ : $F-U$ is linear or nonlinear mapping, it is posed if the problem can satisfy the following 3 conditions:

1) $\forall u \in U$, there is $z \in F$ which can satisfy equation (1)

2) Assuming $u_{1}, u_{2} \in U$, if $z_{1}$ and $z_{2}$ are the solution of equation (1) corresponding to $u_{1} \neq u_{2}$, then $z_{1} \neq z_{2}$

3) The solution is stable comparing to the space (F, U), when $\forall \epsilon>0, \exists \delta(\epsilon)>0$, if

Then

$$
\rho \mathrm{U}\left(u_{1}, u_{2}\right) \leq \delta(\epsilon),\left(u_{1}, u_{2} \in U\right)
$$

$$
\rho \mathrm{F}\left(z_{1} z_{2}\right)<\epsilon,\left(A z_{1}=u_{1}, A z_{2}=u_{2}\right)
$$

The posedness is related to the $\mathrm{A}, \mathrm{F}, \mathrm{U}$, the above three posed conditions have practical background and harsh conditions. In the practical engineering problem, the inverse results are often discrete because of the uncertainty of system, model, and measurement data and so on $[5,6]$.

In the solution of inverse problem, except for the mathematical and physical method to solve the non-posed problem and calculate the stability, neural network is another method. It will not be restrained to the three conditions of applicability, increasing the possibility and acceptability of solving the inverse problem. For the gray or even dark system problem, the neural network method is much easier to approach the solution comparing the complicated numerical method. [7]

The forensic of accident of bridge engineering is a gray inverse problem. It is difficult to build the model which can describe the connection precisely and get the posed solution. Therefore, it is efficient to apply the method in other subject such as neural network method to solve the inverse problem and find the regulation.

\section{Frame Design of Forensic Expert System}

The essence of neural network method is to simulate large bridge project by neural network method based on the historical experimental data [8]. The main purpose is to build the simulated forensic expert experiments. However, if all the forensic need to be rebuilt and practice the suitable network, the forensic may be independent which is not good for maintaining and developing of the network. To provide a more intelligent and friendly forensic platform, considering the neural network as the key model of analysis, all the inverse problems are integrated as a system which can be added, tested, referenced, practiced and calculated. This is the forensic expert system which can be forensic the inverse problem.

Since the forensic expert system takes a long time to collect and manage to be a more mature network, for a long-term management and maintain, the Visual FoxPro is chose to build visual forensic expert system and assist the engineers to collect forensic sample and import forensic data. Moreover, it can be integrated with the neural network tools MATLAB to realize the visualization of the analysis procedure.

The forensic expert system in bridge engineering can be compose of 3 parts:

1) Fundamental data, import and maintain the bridge forensic

2) Neutral network, call the neutral network code, network practice and forensic analysis by the sample data.

3) System maintain and help part, provide the fundamental maintain tools and help instruction for the users.

Among which, fundamental data and neutral network are the key parts. The fundamental data includes the database of common damage, damage registration database, risk database during construction, suggestion database of experts and comprehensive database. 
The neutral network contains neural network training, data input, management of calculated code and neutral network calculation. It is the key part of the forensic expert system which can realize the reasoning and analysis.

The keys of forensic expert system are fundamental data part and neural network part, however, the two parts are based on the VFP and MATLAB language. To build a completed and friendly forensic expert system, it is necessary to call the functions from each other. VPF language is to realize visual expert system interface for the sample collection, completion, registration and input the initial data needed, and therefore the MATLAB neural network practice and detection results can be viewed on the interface directly which is good for searching for users. The MATLAB part can be used for calculation and simulation for neural network, to call for the VPF database, practice and check the sample and conduct the specified forensic. The connection of VPF and MATLAB can be described as:

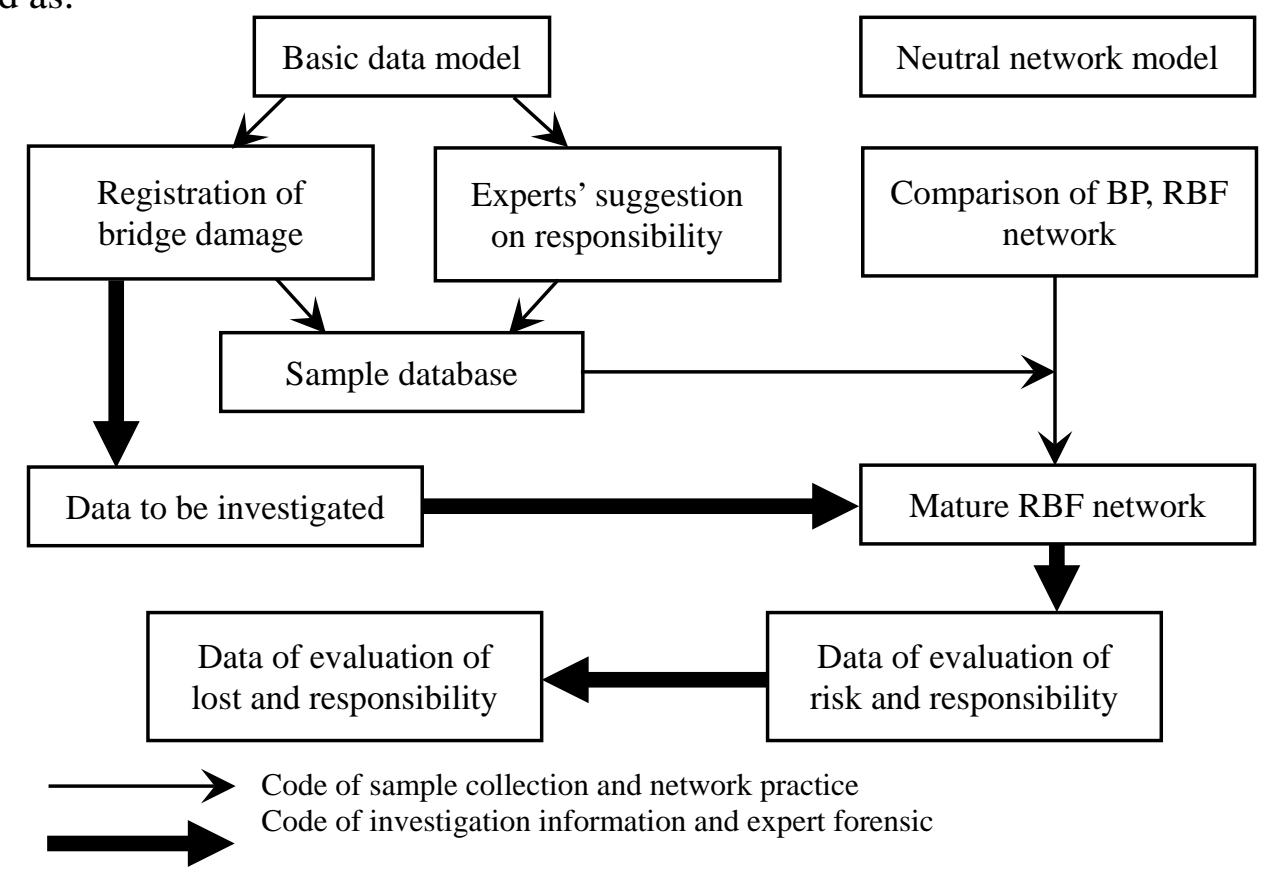

Fig.1 Working relationship between VFP Program and MATLAB Program

\section{Modelling of Systematic Knowledge Database}

This forensic expert system is developed based on the VFP, therefore the fundamental information in the system will exist as fundamental database, and can be saved and updated. The flow chart can be shown as Figure 2:

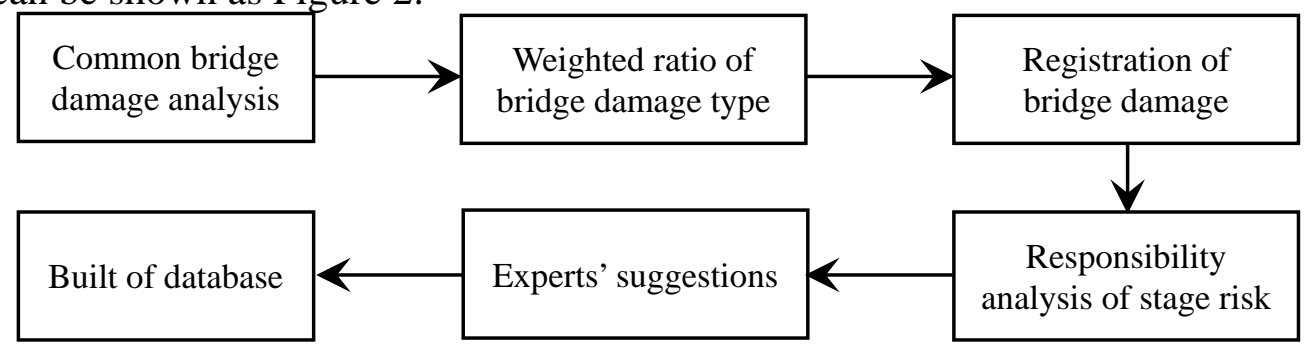

Fig. 2 Process of expert knowledge database establishment

The expert knowledge database is built in the fundamental database, including common damage, damage registration of bridge, staged risk and expert suggestion.

1) The common damage database is mainly used for the management of destruction of different components and the identification of damage in the forensic. Each damage information includes bridge type, structural position, damaged component, importance index, damage type and its impact on accident, type of component, damage phoneme and its influence on the structural health. The database is considered as a reference of identification and evaluation of bridge 
damage. Therefore, the completion of this database can enhance the reliability of expert evaluation, and need to be added and maintained in the long-term forensic.

2) The damage registration database of bridge can be used after the accident. With the registration of professionals on the accident investigation, damage level, damage influence, the damage can be evaluated and quantized suggestions can be provided. The damage registration database of bridge is a collection of the most important bridge damage information. It should be added and updated in the long-term forensic to increase the practice sample and enhance the accuracy of network.

3) Stage risk database is bridge assessment reference of the projecting, design, bidding budget estimate, construction, operation and maintenance, waste removal in the designated period.

4) Expert suggestions database is the evaluation of the accident according to the registration damage database, the possible stage risk in the stage risk database and the damage level and influence, with the consideration of damage mechanism. The database includes the information of damage, responsibility explanation, influence level, and the final ratio of $P_{i}$ responsibility which should follow the equation (4).

$$
P_{i}=\alpha_{i} x_{i} / \sum \alpha_{i} x_{i}
$$

In the equation (4), $x_{i}$ is the contribution level which is defined by the contribution of participants on the accident risk, $\alpha_{i}$ is the weight of contribution

\section{Modelling of Systematic Neutral Network Simulation}

The expert knowledge database needs to be specifically simulated by the intelligent network and the simulation results can be used as expert forensic. Therefore, in the proposed system, two key models and 4 management windows are developed.

1) Window of "neutral network practice" is used for calling functions in MATLAB, practicing the network based on the updated and added basic database of damage registration.

This window can be used to directly call and run the functions which are bridge forensic neutral network codes based on the MATLAB. The neutral network can inherit the expert knowledge database and practice by the sample base to form the mature intelligent network.

The forensic expert system of network and its practice procedure can be seen as figure 3:

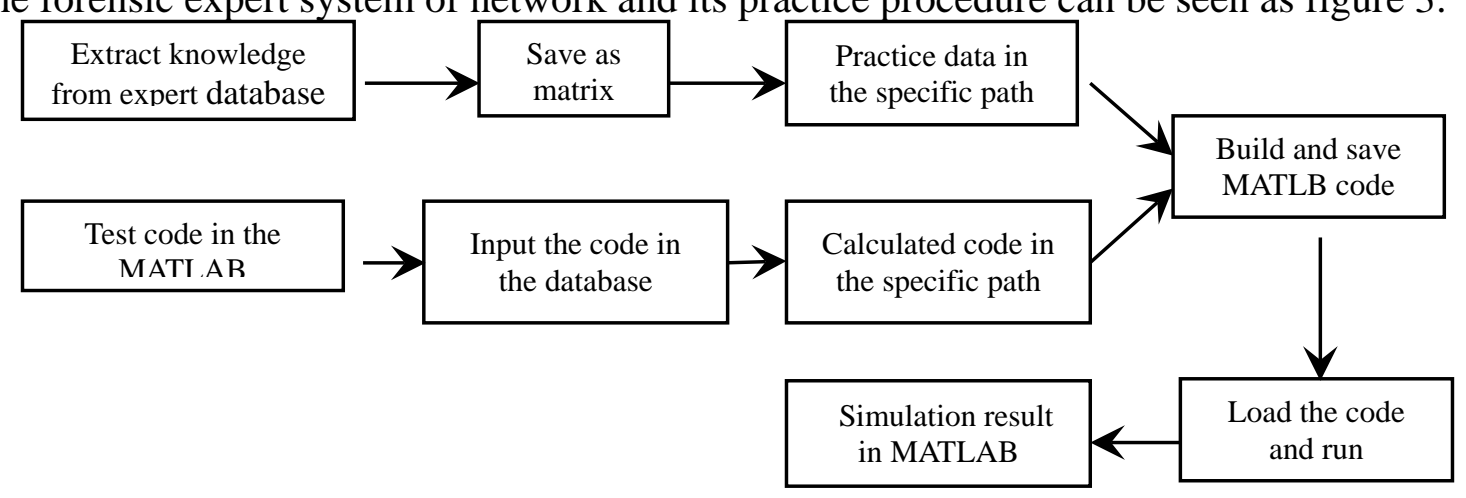

Fig.3 Learning and training process of expert knowledge

2) Window of "input data to be calculated" is used for the input and management of the forensic cases data. It is typically same as the damage registration window of bridge and can be called as "data. txt" form by MATLAB.

3) Window of "management of calculated code" is used for the calling and modification of neutral network in the VFP interface, and can directly test and forensic the system. Taking the analysis accuracy of different cases into consideration, the independent coding window is built for the original code management.

4) Window of "neutral network calculation" is to model and simulate the data for the forensic information to obtain a mature network. It can provide the conclusions of forensic analysis including the components of responsibility, weight and ratio of responsibility, direct economic lost, indirect economic lost and non-economic evaluation. The calculation analysis procedure of neutral network can be seen as Figure 4: 




Fig.4 Bridge damage responsibility analysis process

It can be used for the evaluation analysis of responsibility of bridge damage after validation.

After the investigation of damage, the damage level can be input, managed and forensic in the mature VFP interface. By the intelligent calculation, it can decide the component of risk and provide the suggestion of responsibility ratio based on the reason investigation.

\section{Conclusions}

The specialty of forensic of bridge engineering is analyzed in this paper. As a classical systematical grey inverse problem, the forensic of bridge engineering cannot be analyzed using traditional method because of its unable to solve the posedness. Therefore, the neutral network method is proposed to make the complicated relation as black box and develop the forensic expert system. The neutral network model is built for collection and management of knowledge database based on VFP and MATLAB language. With the combination of the two language, the network can be practiced and optimized by the independent functional windows, and the friendly interface can be built. By the validation, the system can complete the forensic task. By the further data collection and network practice, the forensic accuracy and stability can be increased to ensure that the forensic procedure can be inherited and standard operated.

\section{ACKNOWLEDGEMENT}

The financial support received from the National Natural Science Foundation of China (51541809), Natural Science Foundation of Fujian Province, China (2016J01270) and Scientific and Technological Innovation Platform of Fujian Province, China (2014H2006) are gratefully acknowledged.

\section{REFERENCE}

[1] Guangyuan Huang, Xiaojun Niu, Inverse problem in mathematics and physics [M]. Jinan: the press of Shandong Technology, 1992. (In Chinese)

[2] LIN L, FAN L C, ZHUO W D. Analysis and estimation on risk factors in engineering project life-cycle [C], the 1st International conference on sustainable construction \& risk management. Chongqing, Chongqing Jiaotong University, 2010.

[3] Munan Sun, modification of structure based on neutral network, Shock and Vibration, Vo. 6, 2006

[4] Tingyan Xiao, Shengeng Yu, Yanfei Wang. Solution of inverse problem [M]. Beijing: The press 
of Science, 2003. (In Chinese)

[5] Renwu Wang. SCNN and multidimensional data analysis [M]. Shanghai: the press of Shanghai Academy of Social Sciences, 2008. (In Chinese)

[6] Daoheng Sun, Qiao Hu, Hao Xu, neutral network method of inverse problem in mechanics, Journal of Computation mechanics, Vo. 3, 1996.

[7] Deshan Shan, Dehao Ding, Qiao Li, Zheng Huang, modified model of single cable-stayed bridge based on RBF neutral network [J], Journal of Chongqing Jiaotong University, Vo. 4, 2013.

[8] Lu Zhong, Artificial Neural Network and Its Fusion Applications [M]. Beijing: the press of Science, 2007. (In Chinese) 